\title{
On the Logic of Left-Continuous t-Norms and Right-Continuous t-Conorms
}

\author{
Lluís Godo $^{1(\bowtie)}$, Martín Sócola-Ramos ${ }^{2}$, and Francesc Esteva ${ }^{1}$ \\ 1 Artificial Intelligence Research Institute (IIIA-CSIC), Campus de la UAB, \\ Bellaterra, 08193 Barcelona, Spain \\ \{godo, esteva\}@iiia.csic.es \\ 2 Junivägen 13, 27172 Köpingebro, Sweden \\ martin1992.4@gmail.com
}

\begin{abstract}
Double residuated lattices are expansions of residuated lattices with an extra monoidal operator, playing the role of a strong disjunction operation, together with its dual residuum. They were introduced by Orłowska and Radzikowska. In this paper we consider the subclass of double residuated structures that are expansions of MTLalgebras, that is, prelinear, bounded, commutative and integral residuated lattices. MTL-algebras constitute the algebraic semantics for the MTL logic, the system of mathematical fuzzy logic that is complete w.r.t. the class of residuated lattices on the real unit interval $[0,1]$ induced by left-continuous t-norms. Our aim is to axiomatise the logic whose intended semantics are commutative and integral double residuated structures on $[0,1]$, that are induced by an arbitrary left-continuous t-norm, an arbitrary right-continuous t-conorm, and their corresponding residual operations.
\end{abstract}

Keywords: Mathematical fuzzy logic - Double residuated lattices · MTL $\cdot$ DMCTL $\cdot$ Semilinear logics $\cdot$ Standard completeness

\section{Introduction}

Residuated lattices [7] are a usual choice for general algebras of truth-values in systems of mathematical fuzzy logic [1-3,9], as well as for valuation structures in lattice-valued fuzzy sets (L-fuzzy sets) [8] and in multiple-valued generalisations of information relations $[11,13,14]$. Actually, from this perspective, residuated lattices can be seen as generalizations of the algebraic structures on the real unit interval $[0,1]$ induced by a (left-continuous) t-norm and its residuum. Indeed, in a residuated lattice, besides the lattice-meet $\wedge$ and lattice-join $\vee$ operations, that play the role of the min and max operations in $[0,1]$, there is a monoidal operation $\odot$ that plays the role of a left-continuous t-norm, and its residuum $\rightarrow$ (or its left- and right- residua in case the monoidal operation is not commutative). In a logical setting, while the lattice-meet and lattice-join are used to model a weak conjunction and a weak disjunction respectively, the monoidal operation is 
used to model a strong conjunction connective and its residuum to interpret an implication connective.

However, there is no primitive operation in a residuated lattice that properly accounts for a strong disjunction playing the role of a t-conorm in $[0,1]$. Of course, one can always define a disjunction operation by De Morgan duality from the monoidal operation $\odot$ and the definable residual negation $(\neg x=x \rightarrow 0)$. However, depending on the properties of $\neg$, the resulting disjunction operation may not enjoy nice properties. But even if, for instance, $\neg$ is a well-behaved involutive negation, one is bound to a unique choice for a strong disjunction. To remedy this situation, Orłowska and Radzikowska introduce in [12] more general residuated structures by expanding residuated lattices with an extra monoidal operator playing the role of a t-conorm, together with its (dual) residuum as well. They call them double residuated lattices, DRLs for short.

In [12], the authors study basic properties of DRLs and their application to fuzzy information systems. In this paper we are interested in the particular subclass of double residuated structures that are counterpart of MTL-algebras [6], that is, prelinear, bounded, commutative and integral residuated lattices. MTL-algebras constitute the algebraic semantics for the MTL logic, the system of mathematical fuzzy logic that is complete w.r.t. the class of residuated lattices on the real unit interval $[0,1]$ induced by left-continuous t-norms [10]. Our aim is to axiomatise the logic whose intended semantics are double residuated structures on $[0,1]$, hence induced by an arbitrary pair of a left-continuous tnorm and a right-continuous t-conorm, together with their respective residual operations.

The paper is structured as follows. After this introduction, in Sect. 2 we recall basic definitions and facts about the MTL logic and on double residuated lattices. Then in Sect. 3, as a preliminary step, we axiomatise the logic dual to MTL, called dMTL, while in Sect. 4 we axiomatise the logic complete w.r.t. to the class of double residuated lattices on the real unit interval $[0,1]$. We finish in Sect. 5 with some conclusions and future work. ${ }^{1}$

\section{Preliminaries}

\subsection{A Refresher on MTL}

The language of MTL consists of three binary connectives $\{\wedge, \&, \rightarrow\}$ and a constant $\mathbf{0}$. Formulas are built from a countable set of propositional variables as usual. Further connectives are definable:

$$
\begin{aligned}
& -\neg \varphi:=\varphi \rightarrow \mathbf{0} \\
& -\varphi \vee \psi:=((\varphi \rightarrow \psi) \rightarrow \psi) \wedge((\psi \rightarrow \varphi) \rightarrow \varphi) \\
& -\varphi \equiv \psi:=(\varphi \rightarrow \psi) \&(\psi \rightarrow \varphi) \\
& -\mathbf{1}:=\neg \mathbf{0}
\end{aligned}
$$

We present the MTL axiomatic system proposed in [6]:

\footnotetext{
${ }^{1}$ The results in this paper are based on the Master thesis [15].
} 
(A1) $(\varphi \rightarrow \psi) \rightarrow((\psi \rightarrow \chi) \rightarrow(\varphi \rightarrow \chi))$

(A2) $(\varphi \& \psi) \rightarrow \varphi$

(A3) $(\varphi \& \psi) \rightarrow(\psi \& \varphi)$

(A4) $(\varphi \wedge \psi) \rightarrow \varphi$

(A5) $(\varphi \wedge \psi) \rightarrow(\psi \wedge \varphi)$

$(\mathrm{A} 6)(\varphi \&(\varphi \rightarrow \psi)) \rightarrow(\varphi \wedge \psi)$

$(\mathrm{A} 7)(\varphi \rightarrow(\psi \rightarrow \chi)) \rightarrow((\varphi \& \psi) \rightarrow \chi)$

$(\mathrm{A} 8)((\varphi \& \psi) \rightarrow \chi) \rightarrow(\varphi \rightarrow(\psi \rightarrow \chi))$

$(\mathrm{A} 9)((\varphi \rightarrow \psi) \rightarrow \chi) \rightarrow(((\psi \rightarrow \varphi) \rightarrow \chi) \rightarrow \chi)$

$(\mathrm{A} 10) \mathbf{0} \rightarrow \varphi$

The only inference rule for MTL is Modus Ponens:

$$
(\mathrm{MP}): \frac{\varphi \quad \varphi \rightarrow \psi}{\psi}
$$

MTL is an algebraizable logic and its equivalent algebraic semantics is given by the variety of MTL-algebras. We recall their definition.

Definition 1. A MTL-algebra is a bounded commutative integral residuated lattice

$$
\mathbf{A}=\langle A, \wedge, \vee, \odot, \rightarrow, 0,1\rangle,
$$

where $\wedge, \vee$ are the lattice meet and join operations, and $\langle\odot, \rightarrow\rangle$ is an adjoint pair satisfying the prelinearity condition.

As a direct consequence of its algebraizability, we have the following general completeness result for MTL.

Theorem 1 (General completeness). $\Gamma \vdash_{M T L} \varphi$ iff, for any MTL algebra $\mathbf{A}$ and for every A-evaluation $e, e(\psi)=1$ for all $\psi \in \Gamma$ implies $e(\varphi)=1$.

Thanks to the fact that MTL proves the prelinearity axiom $(\varphi \rightarrow \psi) \vee(\psi \rightarrow$ $\varphi$ ) (a direct consequence of (A9)), the above completeness result can be improved to get completeness with respect to linearly-ordered algebras, or chains.

Theorem 2 (Chain completeness). $\Gamma \vdash_{M T L} \varphi$ iff, for any linearly-ordered $M T L$ algebra A and for every A-evaluation $e, e(\psi)=1$ for all $\psi \in \Gamma$ implies $e(\varphi)=1$.

In other words, MTL is a semilinear logic [4]. As a matter of fact, semilinearity is inherited by many expansions of MTL with new axioms and (finitary) inference rules: the authors of $[4,5]$ prove that an expansion $S$ of MTL is semilinear iff for each newly added finitary inference rule

$$
\text { (R): } \frac{\Gamma}{\varphi},
$$

its corresponding $\vee$-form 
$\left(\mathbf{R}^{\vee}\right): \frac{\Gamma \vee p}{\varphi \vee p}$

is derivable in $S$ as well, where $p$ is an arbitrary propositional variable not appearing in $\Gamma \cup\{\varphi\}$.

Moreover, going back to MTL, to check the validity of a deduction in MTL we can even restrict ourselves to check it on the class of MTL-chains defined on the real unit interval $[0,1]$, also known as standard chains. This was proved by Jenei and Montagna in [10].

Theorem 3 (Standard completeness). $\Gamma \vdash_{M T L} \varphi$ iff, for any standard $M T L$-chain $\mathbf{A}$ and for every A-evaluation $e, e(\psi)=1$ for all $\psi \in \Gamma$ implies $e(\varphi)=1$.

In algebraic terms, this result indicates that the variety of MTL-algebras is generated by its standard chains. Note that if $\mathbf{A}=\langle[0,1], \wedge, \vee, \odot, \rightarrow, 1,0\rangle$ is a MTL-chain, then necessarily $\odot$ is a left-continuous t-norm and $\rightarrow$ is its residuum. This is why MTL is known as the logic of left-continuous t-norms.

\subsection{Double Residuated Lattices}

As mentioned in the introduction, the class of double residuated lattices (DRL) was introduced by Orłowska and Radzikowska in [12], in order to come up with algebraic structures general enough to accommodate on a lattice not only a strong conjunction and its residuum as in the case of residuated lattices, but also a strong disjunction and its residuum.

Although the definition of double residuated lattice in [12] is very general, for the purpose of this paper we will restrict ourselves to the commutative, bounded and integral case.

Definition 2. A (commutative, bounded, integral) double residuated lattice is a structure of the form

$$
\mathbf{A}=\langle A, \wedge, \vee, \odot, \rightarrow, \oplus, \leftarrow, 1,0\rangle
$$

where:

$-\langle A, \wedge, \vee, \odot, \rightarrow, 1,0\rangle$ is a (commutative, bounded, integral) residuated lattice,

$-\langle A, \oplus, 0\rangle$ is a commutative monoid,

$-\leftarrow$ is the dual residuum of $\oplus$, i.e. for all $x, y, z \in A$ the following dual residuation condition holds:

$$
y \leq x \oplus z \quad \text { iff } \quad x \leftarrow y \leq z .
$$

Analogously to (commutative, bounded, integral) residuated lattices, DRLalgebras form a variety. In a DRL-algebra, the lattice order relation can be recovered from both residua, indeed we have:

$$
x \leq y \quad \text { iff } \quad x \rightarrow y=1 \quad \text { iff } \quad y \leftarrow x=0,
$$


and, as a matter of fact, this common underlying order is the only link between both adjoint pairs of operations $(\odot, \rightarrow)$ and $(\oplus, \leftarrow)$.

DMCTL-algebras are also introduced in [12] as the subvariety of DRLalgebras satisfying a pre-linearity condition and its dual version.

Definition 3. Let $\mathbf{A}=\langle A, \wedge, \vee, \odot, \rightarrow, \oplus, \leftarrow, 1,0\rangle$ be a bounded, commutative and integral DRL. Then $\mathbf{A}$ is called:

A double monoidal t-norm logic (DMTL) algebra iff for all $x, y, z \in A$ it satisfies:

$$
(x \rightarrow y) \vee(y \rightarrow x)=1
$$

$A$ double monoidal t-conorm logic (DMCL) algebra iff for all $x, y, z \in A$ it satisfies:

$$
(x \leftarrow y) \wedge(y \leftarrow x)=0
$$

(Dual Prelinearity)

If $\mathbf{A}$ is both a DMTL-and a DMCL-algebra, then it is called a double monoidal t-conorm and t-norm logic (DMCTL) algebra.

Natural (and inspiring) examples of DMCTL-algebras are structures on the unit real interval $[0,1]$ of the form

$$
\langle[0,1], \min , \max , \odot, \rightarrow, \oplus, \leftarrow, 1,0\rangle,
$$

where $\odot$ is a left-continuous t-norm and $\oplus$ is a right-continuous t-conorm. Recall that a t-norm $\oplus$ has residuum iff it is left-continuous, and similarly, a t-conorm $\oplus$ has a dual residuum iff it is right-continuous. In such a case, the residuum of $\odot$ is given by

$$
x \rightarrow y=\max \{z \in[0,1]: x \odot z \leq y\},
$$

and the dual residuum of $\oplus$ is given by

$$
x \leftarrow y=\min \{z \in[0,1]: x \oplus z \geq y\} .
$$

And actually, any DMCTL-algebra on $[0,1]$ is of that form.

\section{3 dMTL: The Dual Logic of MTL}

Before expanding the logic MTL with a strong disjunction and its residuum, it is convenient to start with a dualised version of MTL, that we call dMTL, with only a strong disjunction and its residuum. It is just a formal exercise, but it will help later when defining the whole logic.

The language of dMTL will be built from a countable set of propositional variables and the primitive set of connectives $\{\vee, \odot, \leftarrow\}$ together with the constant 1, as usual: 
- All propositional variables and $\mathbf{1}$ are formulas.

- If $\varphi, \psi$ are formulas, then $\varphi \odot \psi, \varphi \leftarrow \psi$ and $\varphi \vee \psi$ are formulas.

The following connectives are definable:

1. $\vdash \varphi:=\varphi \leftarrow \mathbf{1}$

2. $\varphi \wedge \psi:=((\varphi \leftarrow \psi) \leftarrow \psi) \vee((\psi \leftarrow \varphi) \leftarrow \varphi)$

3. $0:=-1$

The axiom system for dMTL, completely dual to that for MTL, is as follows:

$(\mathrm{dA} 1)(\varphi \leftarrow \psi) \leftarrow((\psi \leftarrow \chi) \leftarrow(\varphi \leftarrow \chi))$

$(\mathrm{dA} 2)(\varphi \odot \psi) \leftarrow \varphi$

$(\mathrm{dA} 3)(\varphi \odot \psi) \leftarrow(\psi \odot \varphi)$

$(\mathrm{dA} 4)(\varphi \vee \psi) \leftarrow \varphi$

$(\mathrm{dA} 5)(\varphi \vee \psi) \leftarrow(\psi \vee \varphi)$

$(\mathrm{dA} 6)(\varphi \odot(\varphi \leftarrow \psi)) \leftarrow(\varphi \vee \psi)$

$(\mathrm{dA} 7)(\varphi \leftarrow(\psi \leftarrow \chi)) \leftarrow((\varphi \odot \psi) \leftarrow \chi)$

$(\mathrm{dA} 8)((\varphi \odot \psi) \leftarrow \chi) \leftarrow(\varphi \leftarrow(\psi \leftarrow \chi))$

$(\mathrm{dA} 9)((\varphi \leftarrow \psi) \leftarrow \chi) \leftarrow(((\psi \leftarrow \varphi) \leftarrow \chi) \leftarrow \chi)$

$(\mathrm{dA} 10) \mathbf{1} \leftarrow \varphi$

The only inference rule for dMTL is the following dual form of Modus Ponens:

$$
(\mathrm{dMP}): \frac{\psi \quad \psi \leftarrow \varphi}{\varphi}
$$

Analogously to MTL, dMTL is also an algebraizable logic, whose equivalent algebraic semantics is given by the class of what we call dMTL-algebras. A $d M T L$-algebra is a structure $\mathbf{A}=\langle A, \wedge, \vee, \oplus, \leftarrow, 0,1\rangle$ such that:

$-\langle A, \wedge, \vee, 0,1\rangle$ is a lattice

- $(A, \oplus, 0)$ is a commutative monoid,

$-\leftarrow$ is the dual residuum of $\oplus$, i.e. for all $x, y, z \in L$ the following dual residuation condition holds:

$$
y \leq x \oplus z \quad \text { iff } \quad x \leftarrow y \leq z .
$$

- The following dual prelinearity condition holds:

$$
(x \leftarrow y) \wedge(y \leftarrow x)=0
$$

In fact, dMTL-algebras are the dual structures of MTL-algebras in the following sense. Let $\langle A, \wedge, \vee, 0,1\rangle$ be a lattice, and consider its dual version $\left\langle A, \wedge^{d}, \vee^{d}, 0^{d}, 1^{d}\right\rangle$, where $\wedge^{d}=\vee, \vee^{d}=\wedge, 0^{d}=1$ and $1^{d}=0$. Then, it is easy to check that $\mathbf{A}=\langle A, \wedge, \vee, \oplus, \leftarrow, 0,1\rangle$ is a dMTL-algebra iff $\mathbf{A}^{d}=\left\langle A, \wedge^{d}, \vee^{d}, \oplus, \leftarrow\right.$ $\left., 0^{d}, 1^{d}\right\rangle$ is a MTL-algebra. In other words, a dMTL-algebra is just a MTL-algebra over its dual lattice reduct.

As a consequence of this observation, it becomes clear that the logic dMTL is not a 1-preserving logic but a 0 -preserving logic. Indeed, all the above axioms are evaluated to 0 by any evaluation on a dMTL-algebra and the inference rule (dMP) does not preserve the truth but the falsity: indeed, for any evaluation $e$, if $e(\psi)=0$ and $e(\psi \leftarrow \varphi)=0$, then $e(\varphi)=0$ as well. 
Theorem 4. For any set of dMTL-formulas $\Gamma \cup\{\varphi\}, \Gamma \vdash_{d M T L} \varphi$ iff, for any $d M T L$ algebra $\mathbf{A}$ and for every $\mathbf{A}$-evaluation $e, e(\psi)=0$ for all $\psi \in \Gamma$ implies $e(\varphi)=0$.

Moreover, one can show that MTL and dMTL are equivalent deductive systems in the following strong sense. Let ${ }^{+}$be the map from dMTL-formulas to MTL-formulas such that

$$
\begin{aligned}
& \mathbf{1}^{+}=\mathbf{0}, p^{+}=p \text {, for each propositional variable } p \text {, and } \\
& (\varphi \vee \psi)^{+}=\varphi^{+} \wedge \psi^{+},(\varphi \leftarrow \psi)^{+}=\varphi^{+} \rightarrow \psi^{+},(\varphi \odot \psi)^{+}=\varphi^{+} \& \psi^{+},
\end{aligned}
$$

and let ${ }^{*}$ be the converse map from MTL to dMT-formulas, i.e. the map such that

$$
\begin{aligned}
& \mathbf{0}^{*}=\mathbf{1}, p^{*}=p \text {, for each propositional variable } p, \text { and } \\
& (\varphi \wedge \psi)^{*}=\varphi^{*} \vee \psi^{*},(\varphi \rightarrow \psi)^{*}=\varphi^{*} \leftarrow \psi^{*},(\varphi \& \psi)^{*}=\varphi^{*} \odot \psi^{*} .
\end{aligned}
$$

Then one can easily prove that deductions can be translated from one logic to the other.

Lemma 1. The following conditions hold:

1. For any set of $M T L$-formulas $\Gamma \cup\{\varphi\}$ : if $\Gamma \vdash_{M T L} \varphi$, then $\Gamma^{*} \vdash_{d M T L} \varphi^{*}$.

2. For any set of dMTL-formulas $\Gamma \cup\{\varphi\}$ : If $\Gamma \vdash_{d M T L} \varphi$, then $\Gamma^{+} \vdash_{M T L} \varphi^{+}$.

\section{The Logic DMCTL: Putting Together MTL and dMTL}

In this section we introduce the double monoidal t-conorm and t-norm logic, DMCTL for short, as the expansion of the logic MTL with a strong disjunction and its dual residuum.

The language of the logic DMCTL expands the language of MTL (built over primitive connectives $\wedge, \&, \rightarrow$ and $\mathbf{0}$ ) with two new connectives $\{\odot, \leftarrow\}$, standing respectively for a strong disjunction and its dual residuum.

We will continue using the following definable connectives:

1. $\neg \varphi:=\varphi \rightarrow \mathbf{0}$

2. $\varphi \vee \psi:=((\varphi \rightarrow \psi) \rightarrow \psi) \wedge((\psi \rightarrow \varphi) \rightarrow \varphi)$

3. $\varphi \equiv \psi:=(\varphi \rightarrow \psi) \&(\psi \rightarrow \varphi)$

4. $\vdash \varphi:=\varphi \leftarrow \mathbf{1}$

From the syntactic point of view, we define the logic DMCTL by expanding MTL with, roughly speaking, the 'negation' of the axioms and rules of dMTL.

Definition 4. DMCTL is the expansion of MTL with the following set of axioms

$$
\{\neg \Phi: \Phi \in\{(d A 1), \ldots,(d A 10)\}\}
$$


and the rules

$$
(\mathrm{R} 1): \frac{\varphi \rightarrow \psi}{\neg(\psi \leftarrow \varphi)} \quad(\mathrm{R} 2): \frac{\neg(\psi \leftarrow \varphi)}{\varphi \rightarrow \psi}
$$

Note that rules $(\mathrm{R} 1)$ and $(\mathrm{R} 2)$ enforce the requirement that the order relations induced by both implications coincide.

Remark 5. Observe that the corresponding 'negation' of the dual of modus ponens ( $\mathrm{dMP})$, i.e. the rule

$$
\frac{\neg \varphi \quad \neg(\varphi \leftarrow \psi)}{\neg \psi}
$$

does not appear in the above definition of the DMCTL logic since this rule is derivable using (R2) and modus tollens (from $\varphi \rightarrow \psi$ and $\neg \psi$, derive $\neg \varphi$ ), that is already derivable in $M T L$, and hence in DMCTL as well.

It is not hard to show that DMCTL enjoys the following congruence properties for the new connectives.

Lemma 2. DMCTL proves the following congruence properties for $\odot$ and $\leftarrow$ :

(i) $\varphi \equiv \psi \vdash_{D M C T L} \varphi \odot \chi \equiv \psi \odot \chi$

(ii) $\varphi \equiv \psi \vdash_{D M C T L}(\psi \leftarrow \chi) \equiv(\varphi \leftarrow \chi)$

(iii) $\varphi \equiv \psi \vdash_{D M C T L}(\chi \leftarrow \varphi) \equiv(\chi \leftarrow \psi)$

These properties point out that DMCTL is in fact a weakly implicative logic and hence algebraizable as well [5], and it has the variety of DMCTL-algebras as its equivalent algebraic semantics. Therefore we already have for free the following general completeness result.

Theorem 6 (General completeness). For any set of DMCTL-formulas $\Gamma \cup$ $\{\varphi\}, \Gamma \vdash_{D M C T L} \varphi$ iff, for any DMCTL-algebra $\mathbf{A}$ and for every $\mathbf{A}$-evaluation $e, e(\psi)=1$ for all $\psi \in \Gamma$ implies $e(\varphi)=1$.

By definition, DMCTL is a expansion of MTL, but in fact we can show more.

Lemma 3. DMCTL is a conservative expansion of $M T L$.

The relation between dMTL and DMCTL is obviously a bit different. In the following, we will use $\vdash_{M T L}^{*}$ and $\vdash_{d M T L}^{*}$ to denote derivations (in the language of DMCTL) using only axioms and rules of MTL and of dMTL respectively. ${ }^{2}$

Lemma 4. For any set of dMTL formulas $\Gamma \cup\{\varphi\}$, we have:

If $\Gamma \vdash_{d M T L}^{*} \varphi$ then $\neg \Gamma \vdash_{D M C T L} \neg \varphi$, where $\neg \Gamma=\{\neg \psi \mid \psi \in \Gamma\}$.

$\overline{2}$ This means that, e.g. in a derivation $\Gamma \vdash_{d M T L}^{*} \varphi$, any maximal subformula in $\Gamma \cup\{\varphi\}$ not belonging to the language of dMTL is treated as a new propositional variable. 


\subsection{DMCTL ${ }^{\ell}$ : The Semilinear Version of DMCTL}

According to the observation made in Sect.2.1, DMCTL, as an expansion of MTL, would be semilinear iff the following $\vee$-forms of the rules (R1) and (R2) following rules

$$
\left(\mathrm{R} 1^{\vee}\right): \frac{(\varphi \rightarrow \psi) \vee \gamma}{\neg(\psi \leftarrow \varphi) \vee \gamma} \quad\left(\mathrm{R} 2^{\vee}\right): \frac{\neg(\psi \leftarrow \varphi) \vee \gamma}{(\varphi \rightarrow \psi) \vee \gamma}
$$

were derivable in DMCTL. However, we have not succeeded in proving this.

This leads us to consider the logic $\mathrm{DMCTL}^{\ell}$ by replacing in DMCTL the rules $(\mathrm{R} 1)$ and $(\mathrm{R} 2)$ by the above stronger two rules $\left(\mathrm{R} 1^{\vee}\right)$ and $\left(\mathrm{R} 2^{\vee}\right)$ respectively. Then, it is clear that $\mathrm{DMCTL}^{\ell}$ is indeed a semilinear logic whose algebraic semantics is given by the quasi-variety of $\mathrm{DMCTL}^{\ell}$-algebras, where a DMCTL ${ }^{\ell}$ algebra is just a DMCTL-algebra satisfying the two following additional condition (in fact it consists of two quasi-equations):

$-(x \rightarrow y) \vee z=1$ iff $\neg(x \leftarrow y) \vee z=1$

Since in a linearly ordered lattice, we have $x \vee y=1$ iff either $x=1$ or $y=1$, an easy observation is that the set of linearly-ordered $\mathrm{DMCTL}^{\ell}$-algebras and linearly-ordered DMCTL-algebras coincide.

Lemma 5. A is a linearly ordered DMCTL $L^{\ell}$-algebra iff $\mathbf{A}$ is a linearly ordered DMCTL-algebra.

Therefore, we have the following chain-completeness result for $\mathrm{DMCTL}^{\ell}$.

Theorem 7 (Chain completeness). The logic DMCTL $L^{\ell}$ is complete with respect to the class of linearly ordered DMCTL-algebras.

In algebraic terms, this result says that the quasivariety of $\mathrm{DMCTL}^{\ell}$-algebras is generated by the class of its linearly-ordered members.

\subsection{Standard Completeness of DMCTL ${ }^{\ell}$}

Finally, in this section we show that, in fact, DMCTL $^{\ell}$ enjoys standard completeness, i.e. completeness with respect to the class of DMCTL-chains on the real unit interval $[0,1]$, or in other words, completeness w.r.t. those algebras on $[0,1]$ defined by a left-continuous t-norm and a right-continuous t-conorm and their residua. To do so, one has to show that if a formula $\varphi$ does not get the value 1 for some evaluation on a countable linearly ordered DMCTL-algebra, then we can always find a linearly ordered DMCTL-algebra on the unit real interval $[0$, 1] and an evaluation $e$ on this algebra such that $e(\varphi)<1$.

Mimicking the constructions in the proof of the standard completeness for MTL in [10], we start with a countable DMCTL-chain

$$
\mathbf{S}=\left\langle S, \odot, \rightarrow, \oplus, \leftarrow, \leq_{S}, 0_{S}, 1_{S}\right\rangle,
$$

and we go through the steps we sketch below: 
Step 1. We densify $\mathbf{S}$ in two different ways:

(a) Define the structure $\mathbf{X}=\left\langle X, \circ, \preceq, 0_{X}, 1_{X}\right\rangle$ as follows:

(i) $X=\{(s, 1): s \in S\} \cup\left\{(s, q) \mid s \in S\right.$ and $\exists s^{\prime}, s=\operatorname{suc}\left(s^{\prime}\right)>s^{\prime}, q \in$ $\mathbb{Q} \cap(0,1)\}$

(ii) $(s, q) \preceq\left(s^{\prime}, q^{\prime}\right)$ iff either $s<_{S} s^{\prime}$, or $s=s^{\prime}$ and $q \leq q^{\prime}$,

(iii) $0_{X}=\left(0_{S}, 1\right)$ and $1_{X}=\left(1_{S}, 1\right)$,

(iv) $\circ$ is a binary operation defined as

$$
(s, q) \circ\left(s^{\prime}, q^{\prime}\right)= \begin{cases}\min _{X}\left\{(s, q),\left(s^{\prime}, q^{\prime}\right)\right\}, & \text { if } s \odot s^{\prime}=\min _{S}\left(s, s^{\prime}\right) \\ \left(s \odot s^{\prime}, 1\right), & \text { otherwise }\end{cases}
$$

where $\min _{X}$ and $\min _{S}$ are the lattice meets with respect to $\preceq$ and $\leq_{S}$ respectively.

(b) Define the structure $\mathbf{Y}=\left\langle Y, \bullet, \preceq, 0_{Y}, 1_{Y}\right\rangle$ as follows:

(i) $Y=\{(s, 0): s \in S\} \cup\{(s, q) \mid s \in S$ and $\exists \operatorname{suc}(s)>s, q \in \mathbb{Q} \cap(0,1)\}$,

(ii) $(s, q) \preceq\left(s^{\prime}, q^{\prime}\right)$ iff either $s<_{S} s^{\prime}$, or $s=s^{\prime}$ and $q \leq q^{\prime}$,

(iii) $0_{Y}=\left(0_{S}, 0\right)$ and $1_{Y}=\left(1_{S}, 0\right)$,

(iv) $\bullet$ is a binary operation defined as

$$
(s, q) \bullet\left(s^{\prime}, q^{\prime}\right)= \begin{cases}\max _{X}\left\{(s, q),\left(s^{\prime}, q^{\prime}\right)\right\}, & \text { if } s \oplus s^{\prime}=\max _{S}\left(s, s^{\prime}\right) \\ \left(s \oplus s^{\prime}, 0\right), & \text { otherwise }\end{cases}
$$

where $\max _{X}$ and $\max _{S}$ are the lattice joins with respect to $\preceq$ and $\leq_{S}$ respectively.

Note that while in $\mathbf{X}$ we have $\lim _{q \rightarrow 1}(s, q)=(s, 1)$ and $\lim _{q \rightarrow 0}(s, q)=$ $\left(s^{\prime}, 1\right)$ whenever $s^{\prime}=\operatorname{suc}(s)<s$, in $\mathbf{Y}$ we have $\lim _{q \rightarrow 1}\left(s^{\prime}, q\right)=(s, 1)$ and $\lim _{q \rightarrow 0}\left(s^{\prime}, q\right)=\left(s^{\prime}, 0\right)$.

Step 2. Although they are different, $\left\langle X, \preceq, 0_{X}, 1_{X}\right\rangle$ and $\left\langle Y, \preceq, 0_{Y}, 1_{Y}\right\rangle$ are order isomorphic through the mapping $h: X \rightarrow Y$ defined as

$$
\left\{\begin{array}{l}
h((s, 1))=(s, 0) \\
h((s, r))=\left(s^{\prime}, r\right) \text { if } r<1 \text { and } s=\operatorname{suc}\left(s^{\prime}\right)
\end{array}\right.
$$

Moreover, $h$ preserves suprema and infima.

Step 3. One can show that $\left\langle X, \circ, \preceq, 0_{X}, 1_{X}\right\rangle$ is a commutative linearly ordered integral monoid with null element $0_{X}$ and that $\circ$ is in fact left-continuous with respect to the order topology on $\langle X, \preceq\rangle$.

Step 4. Analogously, one can show that $\left\langle Y, \bullet, \preceq, 0_{Y}, 1_{Y}\right\rangle$ is a commutative linearly ordered integral monoid with null element $1_{Y}$ and that $\bullet$ is rightcontinuous with respect to the order topology on $\langle Y, \preceq\rangle$.

As a consequence: the structure $\left\langle X, \bullet *, \preceq, 0_{X}, 1_{X}\right\rangle$, where

$$
(s, r) \bullet *\left(s^{\prime}, r^{\prime}\right)=h^{-1}\left(h((s, r)) \bullet h\left(\left(s^{\prime}, r^{\prime}\right)\right)\right),
$$

is a commutative linearly ordered integral monoid with null element $1_{X}$ and show that $\bullet *$ is right-continuous with respect to the order topology on $\langle X, \preceq\rangle$. 
Step 5. The map $\Phi: S \rightarrow X$ defined as $\Phi(s)=(s, 1)$ is an embedding of the structure $\left\langle S, \odot, \oplus, \leq_{S}, 0_{S}, 1_{S}\right\rangle$ into the structure $\left\langle X, \circ, \bullet^{*}, \preceq, 0_{X}, 1_{X}\right\rangle$, and moreover, restricted to $\Phi(S)$ :

(1) The residuum of $\circ$ exists, call it $\Rightarrow$, and $\Phi(s) \Rightarrow \Phi\left(s^{\prime}\right)=\Phi\left(s \rightarrow s^{\prime}\right)$ for all $s, s^{\prime} \in S$.

(2) The residuum of $\bullet *$ exists, call it $\Leftarrow$, and $\Phi^{\prime}(s) \Leftarrow \Phi^{\prime}\left(s^{\prime}\right)=\Phi^{\prime}\left(s \leftarrow s^{\prime}\right)$ for all $s, s^{\prime} \in S$.

Step 6. Since $X$ is countable and dense, the structure $\left\langle X, \circ, \Rightarrow, \bullet *, \Leftarrow, \preceq, 0_{X}, 1_{X}\right\rangle$ is order-isomorphic (through a mapping $\Psi: X \rightarrow \mathbb{Q} \cap[0,1]$ ) to a structure on the rationals in $[0,1],\left\langle\mathbb{Q} \cap[0,1], \circ^{\prime}, \Rightarrow^{\prime}, \bullet^{\prime}, \Leftarrow^{\prime}, \leq, 0,1\right\rangle$.

Step 7. The structure $\left\langle\mathbb{Q} \cap[0,1], \circ^{\prime}, \bullet^{\prime}, \leq, 0,1\right\rangle$ can be embedded into an analogous structure $\langle[0,1], \hat{o}, \hat{\bullet}, \leq, 0,1\rangle$ on the real unit interval, where:

(1) $\hat{o}$ is the extension of $o^{\prime}$ defined by means of left-continuity, i.e. $\alpha \hat{o} \beta=$ $\sup \left\{x \circ^{\prime} y \mid x, y \in \mathbb{Q} \cap[0,1], x \leq \alpha, y \leq \beta\right\}$. Moreover ô is left-continuous.

(2) $\hat{\bullet}$ is the extension of $\bullet$ ' defined by means of right-continuity, i.e. $\alpha \hat{\bullet} \beta=$ $\inf \left\{x \bullet^{\prime} y \mid x, y \in \mathbb{Q} \cap[0,1], x \geq \alpha, y \geq \beta\right\}$. Moreover $\hat{\bullet}$ is right-continuous.

Step 8. Since $\hat{o}$ is left-continuous and $\hat{\bullet}$ is right-continuous on $[0,1]$, their residua and dual residua exist, call them $\Rightarrow_{\hat{o}}$ and $\Leftarrow_{\hat{\bullet}}$ respectively. Then $\left\langle[0,1], \hat{o}, \Rightarrow_{\hat{o}}, \hat{\bullet}, \Leftarrow \hat{\bullet}, \leq, 0,1\right\rangle$ is a linearly ordered DMCTL-algebra where the initial DMCTL-chain $\mathbf{S}$ can be embedded. In particular, if $\Gamma: S \rightarrow[0,1]$ denotes the composition of the maps $\Phi$ and $\Psi$, it can be shown that $\Gamma\left(s \odot s^{\prime}\right)=\Gamma(s) \hat{o} \Gamma\left(s^{\prime}\right), \Gamma\left(s \oplus s^{\prime}\right)=\Gamma(s) \hat{\bullet} \Gamma\left(s^{\prime}\right)$, $\Gamma\left(s \rightarrow s^{\prime}\right)=\Gamma(s) \Rightarrow_{\hat{o}} \Gamma\left(s^{\prime}\right)$, and $\Gamma\left(s \leftarrow s^{\prime}\right)=\Gamma(s) \Leftarrow_{\hat{o}} \Gamma\left(s^{\prime}\right)$ for all $s, s^{\prime} \in S$.

As a direct consequence of the above constructions, we can claim the standard completeness of the logic DMCTL ${ }^{\ell}$.

Theorem 8 (Standard completeness). The logic DMCTL $L^{\ell}$ is standard complete. In other words, for any set of formulas $\Gamma \cup\{\varphi\}, \Gamma \vdash \varphi$ iff, for any standard DMCTL-algebra $\mathbf{A}$ and any $\mathbf{A}$-evaluation $e$, if $e(\psi)=1$ for all $\psi \in \Gamma$, then $e(\varphi)=1$.

Proof. Suppose $\Gamma \forall_{D M C T L^{\ell}} \varphi$, then by the chain-completeness result, there is a countable DMCTL-chain $\mathbf{S}$ and an S-evaluation $e$ that $e(\psi)=1$ for all $\psi \in \Gamma$ while $e(\varphi)<1$. Then, by the above construction, there is a DMCTL-chain on $[0,1]$ where $\mathbf{A}$ can be embedded. Let $h$ be such an embedding and let $e^{\prime}$ the evaluation of variables on $[0,1]$ defined as $e^{\prime}(p)=h(e(p)$ for every propositional variable $p$. Then one still has that $e^{\prime}(\psi)=1$ for all $\psi \in \Gamma$ while $e^{\prime}(\varphi)<1$.

\section{Conclusions}

In this paper we have been concerned with defining a logic complete with respect to the class of double residuated lattices on $[0,1]$ induced by left-continuous tnorms and right-continuous t-conorms. Future work will focus on different extensions of the logic DMCTL ${ }^{\ell}$, counterparts of well-known axiomatic extensions of MTL, like Involutive MTL (IMTL), Hájek's BL logic, or any of their extensions. 
Acknowledgments. Esteva and Godo acknowledge partial support by the Spanish MINECO/FEDER project RASO (TIN2015- 71799-C2-1-P).

\section{References}

1. Cintula, P., Hájek, P., Noguera, C. (eds.): Handbook of Mathematical Fuzzy Logic - Volume 1, Studies in Logic, Mathematical Logic and Foundations, vol. 37. College Publications, London (2011)

2. Cintula, P., Hájek, P., Noguera, C. (eds.): Handbook of Mathematical Fuzzy Logic - Volume 2, Studies in Logic, Mathematical Logic and Foundations, vol. 38. College Publications, London (2011)

3. Cintula, P., Fermüller, C., Noguera, C. (eds.): Handbook of Mathematical Fuzzy Logic - Volume 3, Studies in Logic, Mathematical Logic and Foundations, vol. 58. College Publications, London (2016)

4. Cintula, P., Noguera, C.: Implicational (semilinear) logics I: a new hierarchy. Arch. Math. Log. 49, 417-446 (2010). https://doi.org/10.1007/s00153-010-0178-7

5. Cintula, P., Noguera, C.: A general framework for mathematical fuzzy logic. In: Cintula, P., Hájek, P., Noguera, C. (eds.) Handbook of Mathematical Fuzzy Logic, Vol. 1, Volume 37 of Studies in Logic, Chap. 1, pp. 103-207. College Publications, London (2011)

6. Esteva, F., Godo, L.: Monoidal t-norm based logic: towards a logic for leftcontinuous t-norms. Fuzzy Sets Syst. 124(3), 271-288 (2001)

7. Galatos, N., Jipsen, P., Kowalski, T., Ono, H.: Residuated Lattices: An Algebraic Glimpse at Substructural Logics. Elsevier, Amsterdam (2007)

8. Goguen, J.A.: The logic of inexact concepts. Synthese 19, 325-373 (1968-1969)

9. Hájek, P.: Metamathematics of Fuzzy Logic. Kluwer, Dordrecht (1998)

10. Jenei, S., Montagna, F.: A proof of standard completeness for Esteva and Godo's logic MTL. Stud. Logica 70(2), 183-192 (2002). https://doi.org/10.1023/ A:1015122331293

11. Orłowska, E.: Many-valuedness and uncertainty. In: Multiple-Valued Logic, vol. 4, pp. 207-227 (1999)

12. Orłowska, E., Radzikowska, A.M.: Double residuated lattices and their applications. In: de Swart, H.C.M. (ed.) RelMiCS 2001. LNCS, vol. 2561, pp. 171-189. Springer, Heidelberg (2002). https://doi.org/10.1007/3-540-36280-0_12

13. Radzikowska, A.M., Kerre, E.: A fuzzy generalisation of information relations. In: Orłowska, E., Fitting, M. (eds.) Beyond Two: Theory and Applications of MultipleValued Logics. STUDFUZZ, vol. 114, pp. 287-312. Springer, Heidelberg (2003). https://doi.org/10.1007/978-3-7908-1769-0_13

14. Radzikowska, A.M., Kerre, E.E.: Fuzzy information relations and operators: an Algebraic approach based on residuated lattices. In: de Swart, H., Orłowska, E., Schmidt, G., Roubens, M. (eds.) Theory and Applications of Relational Structures as Knowledge Instruments II. LNCS (LNAI), vol. 4342, pp. 162-184. Springer, Heidelberg (2006). https://doi.org/10.1007/11964810_8

15. Sócola-Ramos, M.: Double residuated lattices. Master thesis, Universitat de Barcelona, September 2019 